\title{
AN INTIAL-VALUE TECHNIQUE FOR SELF-ADJOINT SINGULARLY PERTURBED TWO-POINT BOUNDARY VALUE PROBLEMS
}

\author{
P. PADMAJA \\ Department of Mathematics \\ Prasad V Potluri Siddhartha Institute of Technology \\ Vijayawada-520007, Andhra Pradesh, INDIA \\ E-mail: padmajapodila@gmail.com \\ P. APARNA \\ Department of Mathematics \\ VNR VignanaJyothi Institute of Engineering and Technology \\ Kukatpally, Hyderabad - 500090, INDIA \\ E-mail: aparnapodila@gmail.com \\ R.S.R.GORLA* \\ Department of Mechanical Engineering Cleveland State University \\ Cleveland, USA \\ E-mail: r.gorla@csuohio.edu
}

\begin{abstract}
In this paper, we present an initial value technique for solving self-adjoint singularly perturbed linear boundary value problems. The original problem is reduced to its normal form and the reduced problem is converted to first order initial value problems. This replacement is significant from the computational point of view. The classical fourth order Runge-Kutta method is used to solve these initial value problems. This approach to solve singularly perturbed boundary-value problems is numerically very appealing. To demonstrate the applicability of this method, we have applied it on several linear examples with left-end boundary layer and rightend layer. From the numerical results, the method seems accurate and solutions to problems with extremely thin boundary layers are obtained.
\end{abstract}

Key words: initial value technique, boundary value problem, singular perturbation problem.

\section{Introduction}

The numerical treatment of singular perturbations is far from trivial because of the boundary layer behavior. In recent decades, this has been a field of increasing interest to applied mathematicians and numerical analysts in view of the challenges the problems there pose to researchers. In this section, we present a brief survey of the literature dealing with the numerical treatment of singular perturbation problems.

For a detailed theory and analytical discussion on singular perturbation problems one may refer to the books and high level monographs: O' Malley [1], Nayfeh [2], Kevorkian and Cole [3], Bender and Orszag [4], Van Dyke [5], and Wasow [6].

For a detailed numerical and asymptotic discussion on singular perturbation problems one may refer to the books and high level monographs: Hemker [7], Hemker and Miller [8], Doolan et al. [9], Morton [10].

\footnotetext{
* To whom correspondence should be addressed
} 
In general, a numerical solution of a boundary value problem will be more difficult than a numerical solution of the corresponding initial-value problems. Hence, we always prefer to convert the second-order problems into first order problems. Gasparo and Macconi [11-14] developed initial value methods for singularly perturbed two-point boundary value problems. Parallel initial value algorithms are developed for singularly perturbed boundary value problems by them.

Reddy and Chakravarthy [15] proposed a method of reduction of order for solving singularly perturbed two point boundary value problems. The solution of the given two-point boundary value problem is numerically computed by solving two suitable initial-value problems easily deduced from the original problem. The method is very easy to implement and is tested on several linear, non-linear problems. They [16] proposed an initial value technique for solving singularly perturbed two point boundary value problems.

Mishra et al. [17] studied an initial value technique for self adjoint singular perturbation boundary value problems. Natesan and Ramanujam [18] studied initial-value technique for singularly perturbed boundary-value problems for second-order ordinary differential equations arising in chemical reactor theory. An initial-value technique is presented for solving singularly perturbed two-point boundary-value problems for linear and semi linear second-order ordinary differential equations arising in chemical reactor theory. The required approximate solution is obtained by combining solutions of two terminal-value problems and one initial-value problem which are obtained from the original boundary-value problem through asymptotic expansion procedures. Error estimates for approximate solutions are obtained. The initial-value technique has been applied to solve various singularly perturbed boundary value problems for second-order ordinary differential equations subject to Dirichlet-type boundary conditions.

Here, we present an initial value technique based on in [17] for solving self-adjoint singularly perturbed linear boundary value problems. The original problem is reduced to its normal form and the reduced problem is converted to first order initial value problems. This replacement is significant from the computational point of view. The classical fourth order Runge-Kutta method is used to solve these initial value problems. This approach to solve singularly perturbed boundary-value problems is numerically very appealing. To demonstrate the applicability of this method, we have applied it on several linear examples with left-end boundary layer and right-end layer. From the numerical results, the method seems accurate and solutions to problems with extremely thin boundary layers are obtained.

\section{Statement of the problem} problem

We consider the following class of a self adjoint singularly perturbed two-point boundary value

$$
L y \equiv-\varepsilon\left(a(x) y^{\prime}(x)\right)^{\prime}+b(x) y(x)=f(x) \quad \text { where } 0 \leq x \leq 1
$$

subject to $\quad y(0)=\alpha, \quad y(l)=\beta$

where $\alpha, \beta \epsilon R, \varepsilon$ is a small positive parameter and $a(x), b(x)$ and $f(x)$ are smooth functions and satisfy

$$
a(x) \geq a^{*}>0, a^{\prime}(x) \geq 0, b(x) \geq b^{*}>0 \text {. }
$$

Under these conditions the operator $L$ admits the maximum principle.

\subsection{Description of the method}

To describe the method, we consider the singularly perturbed two-point boundary -value problem

$$
-\varepsilon\left(a(x) y^{\prime}(x)\right)^{\prime}+b(x) y(x)=f(x) \text { for } 0 \leq x \leq 1,
$$

with boundary conditions 


$$
\begin{aligned}
& y(0)=\alpha, \\
& y(1)=\beta
\end{aligned}
$$

where $\varepsilon$ is a small positive parameter $(0<\varepsilon \ll 1), \alpha, \beta$ are given constants, and the functions $a(x), b(x)$ and $f(x)$ are assumed to be sufficiently smooth in $[0,1]$.

We also suppose that $a(x), b(x)$ satisfies

$$
a(x) \geq a^{*}>0, \quad a^{\prime}(x) \geq 0, \quad b(x) \geq b^{*}>0 .
$$

Equation (2.1) can be rewritten as

$$
\begin{aligned}
& -\varepsilon a(x) y^{\prime \prime}(x)-\varepsilon a^{\prime}(x) y^{\prime}(x)+b(x) y(x)=f(x), \\
& y^{\prime \prime}(x)+\frac{a^{\prime}(x)}{a(x)} y^{\prime}(x)-\frac{b(x)}{\varepsilon a(x)} y(x)=-\frac{f(x)}{\varepsilon a(x)}, \\
& y^{\prime \prime}(x)+P(x) y^{\prime}(x)+Q(x) y(x)=R(x)
\end{aligned}
$$

or

where

$$
\begin{aligned}
& P(x)=\frac{a^{\prime}(x)}{a(x)}, \\
& Q(x)=\frac{-b(x)}{\varepsilon a(x)},
\end{aligned}
$$

and

$$
R(x)=\frac{-f(x)}{\varepsilon a(x)} .
$$

In general, finding the numerical solution of a second-order boundary value problem with $y^{\prime}$ term is more difficult as compared to a second-order boundary value problem without $y^{\prime}$ term. Therefore it is better to convert the second-order boundary-value problem without $y^{\prime}$ term to its normal form.

Now we use the transformation

$$
y(x)=U(x) V(x),
$$

to reduce Eq.(2.1) to its normal form.

Differentiating Eq.(2.4), we get

$$
\left.y^{\prime}(x)=U(x) V^{\prime} X\right)+V(x) U^{\prime}(x) .
$$

Again differentiating above equation, we get 


$$
y^{\prime \prime}(x)=U(x) V^{\prime \prime}(x)+V^{\prime}(x) U^{\prime}(x)+V(x) U^{\prime \prime}(x)+V^{\prime}(x) U^{\prime}(x),
$$

i.e.,

$$
y^{\prime \prime}(x)=U(x) V^{\prime \prime}(x)+2 V^{\prime}(x) U^{\prime}(x)+V(x) U^{\prime \prime}(x),
$$

substituting these in Eq.(2.3), we get

$$
\begin{aligned}
& \left.\left[U(x) V^{\prime \prime}(x)+2 V^{\prime}(x) U^{\prime}(x)+V(x) U^{\prime \prime}(x)\right]+P(x)\left[U(x) V^{\prime} X\right)+V(x) U^{\prime}(x)\right]+ \\
& +Q(x)[U(x) V(x)=R(x),
\end{aligned}
$$

i.e.,

$$
\begin{aligned}
& U(x) V^{\prime \prime}(x)+\left[U^{\prime \prime}(x)+P(x) U^{\prime}(x)+Q(x) U(x)\right] V(x)+ \\
& +\left[2 V^{\prime}(x) U^{\prime}(x)+P(x) U(x) V^{\prime}(x)\right]=R(x), \\
& V^{\prime \prime}(x)+\frac{U^{\prime \prime}(x)+P(x) U^{\prime}(x)+Q(x) U(x)}{U(x)} V(x)=
\end{aligned}
$$

or

$$
=\left[\frac{R(x)-\left[2 V^{\prime}(x) U^{\prime}(x)+P(x) U(x) V^{\prime}(x)\right]}{U(x)}\right],
$$

i.e., $\quad V^{\prime \prime}(x)+A(x) V(x)=H(x)$

where

$$
A(x)=\frac{U^{\prime \prime}(x)+P(x) U^{\prime}(x)+Q(x) U(x)}{U(x)},
$$

and

$$
H(x)=\frac{R(x)-\left[2 V^{\prime}(x) U^{\prime}(x)+P(x) U(x) V^{\prime}(x)\right]}{U(x)} .
$$

we choose

$$
U(x)=\exp \left(-\frac{1}{2} \int_{0}^{x} P(s) d s\right)
$$

differentiating Eq.(2.6), we get

$$
\begin{aligned}
& U^{\prime}(x)=\frac{d}{d x} \exp \left(-\frac{1}{2} \int_{0}^{x} P(s) d s\right)=\exp \left(-\frac{1}{2} \int_{0}^{x} P(s) d s\right) \frac{d}{d x}\left(-\frac{1}{2} \int_{0}^{x} P(s) d s\right) \\
& =\exp \left(-\frac{1}{2} \int_{0}^{x} P(s) d s\right)\left[\frac{-P(x)}{2}\right] .
\end{aligned}
$$

Again differentiating Eq.(2.6a) with respect to $x$, we get 


$$
U^{\prime \prime}(x)=\left(\frac{[P(x)]^{2}}{4}\right) \exp \left(-\frac{1}{2} \int_{0}^{x} P(s) d s\right)-\left(\frac{P^{\prime}(x)}{2}\right) \exp \left(-\frac{1}{2} \int_{0}^{x} P(s) d s\right)
$$

Equation (2.5a) becomes

$$
A(x)=Q(x)-\frac{P^{\prime}(x)}{2}-\frac{[P(x)]^{2}}{4},
$$

Equation (2.5b) becomes

$$
\begin{aligned}
& H(x)=\frac{R(x)-V^{\prime}(x)}{U(x)}\left\{2 \exp \left(-\frac{1}{2} \int_{0}^{x} P(s) d s\right)\left[\frac{-P(x)}{2}\right]+P(x) \exp \left(-\frac{1}{2} \int_{0}^{x} P(s) d s\right)\right\}= \\
& =\frac{R(x)}{U(x)}-\frac{V^{\prime}(x)}{U(x)}\left\{-2\left[\frac{P(x)}{2}\right] \exp \left(-\frac{1}{2} \int_{0}^{x} P(s) d s\right)+P(x) \exp \left(-\frac{1}{2} \int_{0}^{x} P(s) d s\right)\right\}
\end{aligned}
$$

i.e., $\quad H(x)=\frac{R(x)}{U(x)}=\frac{R(x)}{\exp \left(-\frac{1}{2} \int_{0}^{x} P(s) d s\right)}$,

$$
H(x)=R(x) \exp \left(\frac{1}{2} \int_{0}^{x} P(s) d s\right)
$$

We have

$$
\begin{aligned}
& V(0)=\frac{y(0)}{U(0)}=\gamma \quad \text { (say), } \\
& V(1)=\frac{y(1)}{U(1)}=\delta \quad \text { (say) } \quad x \epsilon(0,1) \quad \text { and } \quad \gamma, \delta \epsilon R .
\end{aligned}
$$

On multiplying Eq.(2.5) by $-\varepsilon$, we get

$$
L_{1} V(x)=-\varepsilon V^{\prime \prime}(x)-\varepsilon A(x) V(x)=-\varepsilon H(x),
$$

i.e.,

$$
L_{l} V(x)=-\varepsilon V^{\prime \prime}(x)+W(x) V(x)=Z(x)
$$

with boundary conditions

$$
V(0)=\gamma, \quad V(1)=\delta
$$

where

$$
W(x)=-\varepsilon A(x), \quad Z(x)=-\varepsilon H(x), \quad W(x) \geq W>0 .
$$


In order to obtain the solution $V(x)$ of the problem, let $V_{R}(x)$ be the solution of the reduced problem obtained by putting $\varepsilon=0$ in Eq.(2.8) and by neglecting both boundary conditions.

We have

$$
W(x) V_{R}(x)=Z(x) x \in[0,1]
$$

where

$$
V_{R}(x)=\frac{Z(x)}{W(x)} \quad x \in[0,1]
$$

The asymptotic solution of Eq.(2.8) with the conditions (2.9) is given by

$$
\begin{aligned}
& V(x)=V_{R}(x)+\left\{\left(\gamma-V_{R}(0)\right)(W(0) / W(x))^{1 / 4} \exp \left(\left(-\int_{0}^{x} \sqrt{-W(s) / \varepsilon} d s\right)\right)\right\}+ \\
& +\left\{\left(\gamma-V_{R}(1)\right)(W(1) / W(x))^{1 / 4} \exp \left(-\int_{x}^{1} \sqrt{-W(s) / \varepsilon} d s\right)\right\}
\end{aligned}
$$

where $V_{1}(x)$ and $V_{2}(x)$ are defined on $[0,1]$ by

$$
\begin{gathered}
V_{1}(x)=\exp \left(-\int_{0}^{x} \sqrt{-W(s) / \varepsilon} d s\right), \\
V_{2}(x)=\exp \left(-\int_{x}^{1} \sqrt{-W(s) / \varepsilon} d s\right) .
\end{gathered}
$$

Differentiating Eq.(2.11), we get

$$
V_{l}^{\prime}(x)=\frac{d}{d x}\left\{\exp \left(-\int_{0}^{x} \sqrt{-W(s) / \varepsilon} d s\right)\right\}
$$

i.e.,

$$
\begin{aligned}
& V_{l}^{\prime}(x)=\exp \left(-\int_{0}^{x} \sqrt{-W(s) / \varepsilon} d s\right) \frac{d}{d x}\left(-\int_{0}^{x} \sqrt{-W(s) / \varepsilon} d s\right)= \\
& =\exp \left(-\int_{0}^{x} \sqrt{-W(s) / \varepsilon} d s\right)\left\{-\sqrt{-W(x) / \varepsilon}\left(\frac{1}{\sqrt{\varepsilon}}\right)\right\}, \\
& \sqrt{\varepsilon} V_{l}^{\prime}(x)+\exp \left(-\int_{0}^{x} \sqrt{-W(s) / \varepsilon} d s\right) \sqrt{-W(x) / \varepsilon}=0,
\end{aligned}
$$

using Eq.(2.11), we get 


$$
\begin{aligned}
& \sqrt{\varepsilon} V_{1}^{\prime}(x)+V_{1}(x) \sqrt{-W(x) / \varepsilon}=0 x \in[0,1] \\
& V_{l}(0)=1 .
\end{aligned}
$$

Now differentiating Eq.(2.12), we get

$$
\begin{aligned}
& V_{2}^{\prime}(x)=\frac{d}{d x}\left\{\exp \left(-\int_{x}^{1} \sqrt{-W(s) / \varepsilon} d s\right)\right\}=\exp \left(-\int_{x}^{1} \sqrt{-W(s) / \varepsilon} d s\right) \frac{d}{d x}\left(-\int_{x}^{1} \sqrt{-W(s) / \varepsilon} d s\right)= \\
& =\exp \left(-\int_{x}^{1} \sqrt{-W(s) / \varepsilon} d s\right)\left(\sqrt{-W(s) / \varepsilon}\left(\frac{1}{\sqrt{\varepsilon}}\right)\right), \\
& \sqrt{\varepsilon} V_{2}^{\prime}(x)-\exp \left(-\int_{x}^{1} \sqrt{-W(s) / \varepsilon} d s\right) \sqrt{-W(s) / \varepsilon}=0,
\end{aligned}
$$

using Eq.(2.12), we get

$$
\begin{aligned}
& \sqrt{\varepsilon} V_{2}^{\prime}(x)-V_{2}(x) \sqrt{-W(x) / \varepsilon}=0 x \in[0,1], \\
& V_{2}(0)=1
\end{aligned}
$$

i.e., clearly $V_{l}(x)$ can be found as the solution of the initial -value problem

$$
\begin{aligned}
& \sqrt{\varepsilon} V_{1}^{\prime}(x)+V_{1}(x) \sqrt{-W(x) / \varepsilon}=0 x \in[0,1], \\
& V_{1}(0)=1
\end{aligned}
$$

and $V_{2}(x)$ can be found as the solution of the initial -value problem

$$
\begin{aligned}
& \sqrt{\varepsilon} V_{2}^{\prime}(x)-V_{2}(x) \sqrt{-W(x) / \varepsilon}=0 x \in[0,1], \\
& V_{2}(0)=1 .
\end{aligned}
$$

Now we write the solution as

$$
\begin{aligned}
& V(x)=V_{R}(x)+\left\{\left(\gamma-V_{R}(0)\right)(W(0) / W(x))^{1 / 4} V_{1}(x)\right\}+ \\
& +\left\{\left(\gamma-V_{R}(1)\right)(W(1) / W(x))^{1 / 4} V_{2}(x)\right\}+O(\sqrt{\varepsilon)} .
\end{aligned}
$$

We can approximate the solution of the problem (2.8) by combining the function $V_{R}(x)$ given by (2.10a) with the solution of the initial-value problem (2.13)-(2.14) and (2.15)-(2.16).

Hence the solution of Eq.(2.1) is given by 


$$
y(x)=U(x) V(x)
$$

The whole method is extremely simple to implement. To this end, it is enough to use suitable codes for initial-value problems taking into account that the solution of Eqs (2.13) and (2.15) may change character in the interval of integration. In fact, these problems are generally non-stiff near the starting point, but we must expect stiffness as the integration goes to the end of the interval.

So, the numerical solution of these problems requires a scheme automatically determining whether the problem can be solved more efficiently using a class of methods designed for non-stiff problems or a class of methods suitable for stiff problem.

\section{Numerical examples}

To demonstrate the applicability of the methods, we applied the present method to three singular perturbation problems. These examples have been chosen because either analytic or approximate solutions are available for comparison.

Examples 3.1: Consider the following equation

$$
-\varepsilon\left(y^{\prime}(x)\right)^{\prime}+y(x)=-\cos ^{2}(\pi x)-2 \varepsilon \pi^{2} \cos (2 \pi x),
$$

i-th boundary conditions

$$
\begin{aligned}
& y(0)=0, \\
& y(1)=0,
\end{aligned}
$$

which has the exact solution

$$
y(x)=\frac{\left[\exp \left(\frac{-1(1-x)}{\sqrt{x}}\right)+\exp \left(\frac{-x}{\sqrt{\varepsilon}}\right)\right]}{\left[1+\exp \left(\frac{-1}{\sqrt{\varepsilon}}\right)\right]-\cos ^{2}(\pi x)}
$$

now Eq.(2.17) becomes

$$
\begin{aligned}
& -\varepsilon y^{\prime \prime}(x)+y(x)=-\cos ^{2}(\pi x)-2 \varepsilon \pi^{2} \cos (2 \pi x), \\
& y^{\prime \prime}(x)-\frac{y(x)}{\varepsilon}=\frac{\cos ^{2}(\pi x)}{\varepsilon}+2 \pi^{2} \cos (2 \pi x),
\end{aligned}
$$

by comparing Eqs (2.1) and (2.3), we have

$$
\begin{aligned}
& a(x)=1, \\
& b(x)=1,
\end{aligned}
$$




$$
\begin{aligned}
& f(x)=-\cos ^{2}(\pi x)-2 \varepsilon \pi^{2} \cos (2 \pi x), \\
& P(x)=0, \\
& Q(x)=\frac{-1}{\varepsilon}, \\
& R(x)=\frac{\cos ^{2}(\pi x)-2 \varepsilon \pi^{2} \cos (2 \pi x)}{\varepsilon},
\end{aligned}
$$

also we have

$$
\begin{aligned}
& A(x)=\frac{-1}{\varepsilon}-\frac{1}{2}(0)-\frac{1}{4}(0), \\
& A(x)=\frac{-1}{\varepsilon},
\end{aligned}
$$

from Eq.(2.7), we have

$$
\begin{aligned}
& H(x)=\frac{\cos ^{2}(\pi x)-2 \varepsilon \pi^{2} \cos (2 \pi x)}{\varepsilon} \exp \left(\frac{1}{2} \int_{0}^{x}(0) d s\right), \\
& =\frac{\cos ^{2}(\pi x)-2 \varepsilon \pi^{2} \cos (2 \pi x)}{\varepsilon}\left(e^{0}\right), \\
& \text { i.e., } \quad H(x)=\frac{\cos ^{2}(\pi x)-2 \varepsilon \pi^{2} \cos (2 \pi x)}{\varepsilon},
\end{aligned}
$$

from Eq.(2.6), we have

$$
\begin{aligned}
& U(x)=\exp \left(-\frac{1}{2} \int_{0}^{x}(0) d s\right), \\
& U(x)=e^{0}, \\
& U(x)=1, \\
& V(0)=0 \\
& V(1)=0,
\end{aligned}
$$

Equation (3.3) can be written as 


$$
-\varepsilon V^{\prime \prime}(x)+V(x)=-\cos ^{2}(\pi x)-2 \varepsilon \pi^{2} \cos (2 \pi x)
$$

with boundary conditions

$$
\begin{aligned}
& V(0)=0, \\
& V(1)=0,
\end{aligned}
$$

as $\varepsilon \rightarrow 0$ Eq.(3.8) becomes

$$
\begin{aligned}
& V_{R}(x)=-\cos ^{2}(\pi x)-2 \varepsilon \pi^{2} \cos (2 \pi x), \\
& V_{R}(0)=-1-2 \varepsilon \pi^{2}
\end{aligned}
$$

and

$$
V_{R}(1)=-\cos ^{2}(\pi)-2 \varepsilon \pi^{2} \cos (2 \pi) .
$$

Equation (2.8) becomes

$$
\begin{aligned}
& W(x)=-\varepsilon\left(\frac{-1}{\varepsilon}\right), \\
& W(x)=1
\end{aligned}
$$

now Equation (2.11) becomes

$$
V_{l}(x)=\exp \left(-\int_{0}^{x} \sqrt{\left(\frac{-1}{\varepsilon}\right)} d s\right),
$$

differentiating Eq.(3.14), we get

$$
\begin{aligned}
& V_{l}^{\prime}(x)=\frac{d}{d s}\left\{\exp \left(-\int_{0}^{x} \sqrt{\left(\frac{-1}{\varepsilon}\right)} d s\right)\right\}, \\
& =\exp \left(-\int_{0}^{x} \sqrt{\left(\frac{-1}{\varepsilon}\right)} d s\right) \frac{d}{d s}\left(-\int_{0}^{x} \sqrt{\left(-\frac{1}{\varepsilon}\right)} d s\right), \\
& V_{l}^{\prime}(x)=\exp \left(-\int_{0}^{x} \sqrt{\left(\frac{-1}{\varepsilon}\right)} d s\right)\left(\frac{-1}{\sqrt{\varepsilon}}\right) .
\end{aligned}
$$

The first initial-value problem is given by

$$
V_{l}^{\prime}(x)=V_{l}(x)\left(\frac{-1}{\sqrt{\varepsilon}}\right)
$$

with

$$
V_{1}(0)=1
$$

now Eq.(2.12) becomes 


$$
V_{2}(x)=\exp \left(-\int_{x}^{1} \sqrt{\left(\frac{-1}{\varepsilon}\right)} d s\right) .
$$

The second initial-value problem is given by

$$
V_{2}^{\prime}(x)=V_{2}(x)\left(\frac{1}{\sqrt{\varepsilon}}\right)
$$

with

$$
V_{2}(1)=1
$$

Table 1. The maximum absolute errors for example 3.1 for $\varepsilon=10^{-4}, h=10^{-3}$.

\begin{tabular}{|c|c|c|c|}
\hline$x$ & $y(x)$ & Exact solution & Max. Absolute error \\
\hline 0.0000 & 0.0000000 & 0.0000000 & 0.0000000 \\
\hline 0.0010 & -0.0953248 & -0.0951536 & 0.0001712 \\
\hline 0.0020 & -0.1815593 & -0.1812333 & 0.0003260 \\
\hline 0.0030 & -0.2595668 & -0.2591008 & 0.0004660 \\
\hline 0.0040 & -0.3301286 & -0.3295360 & 0.0005926 \\
\hline 0.0050 & -0.3939515 & -0.3932444 & 0.0007071 \\
\hline 0.0200 & -0.8626122 & -0.8610691 & 0.0015432 \\
\hline 0.0400 & -0.9690687 & -0.9673533 & 0.0017154 \\
\hline 0.0600 & -0.9671498 & -0.9654697 & 0.0016801 \\
\hline 0.0800 & -0.9447580 & -0.9431620 & 0.0015960 \\
\hline 0.1000 & -0.9141079 & -0.9126224 & 0.0014855 \\
\hline 0.2000 & -0.6818311 & -0.6811789 & 0.0006523 \\
\hline 0.4000 & -0.1299758 & -0.1313031 & 0.0013273 \\
\hline 0.6000 & -0.0500067 & -0.0516208 & 0.0016142 \\
\hline 0.8000 & -0.5439070 & -0.5437495 & 0.0001575 \\
\hline 1.0000 & 0.0000000 & 0.0000000 & 0.0000000 \\
\hline
\end{tabular}

Table 2. The maximum absolute errors for example 3.1 for $\varepsilon=10^{-5}, h=10^{-3}$.

\begin{tabular}{|c|c|c|c|}
\hline$x$ & $y(x)$ & Exact solution & Max. Absolute error \\
\hline 0.0000 & 0.0000000 & 0.0000000 & 0.0000000 \\
\hline 0.0010 & -0.2711214 & -0.2710976 & 0.0000238 \\
\hline 0.0020 & -0.4687263 & -0.4686784 & 0.0000479 \\
\hline 0.0030 & -0.6127388 & -0.6126685 & 0.0000703 \\
\hline 0.0040 & -0.7176820 & -0.7175916 & 0.0000904 \\
\hline 0.0050 & -0.7941419 & -0.7940344 & 0.0001076 \\
\hline 0.0200 & -0.9947897 & -0.9946126 & 0.0001771 \\
\hline 0.0400 & -0.9858406 & -0.9856658 & 0.0001748 \\
\hline 0.0600 & -0.9681169 & -0.9679484 & 0.0001685 \\
\hline 0.0800 & -0.9436571 & -0.9434975 & 0.0001596 \\
\hline 0.1000 & -0.9128163 & -0.9126678 & 0.0001485 \\
\hline 0.2000 & -0.6812441 & -0.6811789 & 0.0000652 \\
\hline 0.4000 & -0.1311704 & -0.1313031 & 0.0001327 \\
\hline 0.6000 & -0.0514594 & -0.0516208 & 0.0001614 \\
\hline 0.8000 & -0.5437653 & -0.5437495 & 0.0000158 \\
\hline 1.0000 & 0.0000000 & 0.0000000 & 0.0000000 \\
\hline
\end{tabular}




\section{Example 3.2}

Consider the following equation

$$
-\varepsilon\left(y^{\prime}(x)\right)^{\prime}+(1+x) y(x)=-40\left[x\left(x^{2}-1\right)-2 \varepsilon\right],
$$

with boundary conditions

$$
\begin{aligned}
& y(0)=0, \\
& y(1)=0,
\end{aligned}
$$

which has the exact solution

$$
y(x)=40 x(1-x)
$$

from Eq.(3.18) we have

$$
-\varepsilon y^{\prime \prime}(x)+(1+x) y(x)=-40\left[x\left(x^{2}-1\right)-2 \varepsilon\right] .
$$

This can be rewritten as

$$
y^{\prime \prime}(x)-\frac{(1+x)}{\varepsilon} y(x)=\frac{40\left[x\left(x^{2}-1\right)-2 \varepsilon\right]}{\varepsilon},
$$

comparing Eq.(2.1) and Eq.(2.3), we get

$$
\begin{aligned}
& a(x)=1, \\
& b(x)=(1+x), \\
& f(x)=-40\left[x\left(x^{2}-1\right)-2 \varepsilon\right], \\
& P(x)=0 \\
& Q(x)=\frac{-(1+x)}{\varepsilon} \\
& R(x)=\frac{40\left[x\left(x^{2}-1\right)-2 \varepsilon\right]}{\varepsilon},
\end{aligned}
$$

also we have $\quad A(x)=\frac{-(1+x)}{\varepsilon}-\frac{1}{2}(0)-\frac{1}{4}(0)$ 
i.e., $\quad A(x)=\frac{-(1+x)}{\varepsilon}$,

from Eq.(2.7), we have

$$
\begin{aligned}
& H(x)=\frac{40\left[x\left(x^{2}-1\right)-2 \varepsilon\right]}{\varepsilon} \exp \left(\frac{1}{2} \int_{0}^{x}(0) d s\right) \\
& =\frac{40\left[x\left(x^{2}-1\right)-2 \varepsilon\right]}{\varepsilon} e^{0}, \\
& \text { i.e., } \quad H(x)=\frac{40\left[x\left(x^{2}-1\right)-2 \varepsilon\right]}{\varepsilon},
\end{aligned}
$$

from Eq.(2.6) we have

$$
\begin{aligned}
& U(x)=\exp \left(-\frac{1}{2} \int_{0}^{x}(0) d s\right) \\
& =e^{0} \\
& U(x)=1
\end{aligned}
$$

and

$$
\begin{aligned}
& V(0)=0, \\
& V(1)=0
\end{aligned}
$$

Eq.(3.20) can be written as

$$
-\varepsilon V^{\prime \prime}(x)+(1+x) V(x)=-40\left[x\left(x^{2}-1\right)-2 \varepsilon\right],
$$

with boundary condition

$$
\begin{aligned}
& V(0)=0, \\
& V(1)=0,
\end{aligned}
$$

as $\varepsilon \rightarrow 0$ Eq.(3.25) becomes

$$
\begin{aligned}
& (1+x) V_{R}(x)=-40\left[x\left(x^{2}-1\right)-2 \varepsilon\right], \\
& V_{R}(x)=\frac{-40\left[x\left(x^{2}-1\right)-2 \varepsilon\right]}{(1+x)},
\end{aligned}
$$


hence

$$
\begin{aligned}
& V_{R}(0)=\frac{-40(-2 \varepsilon)}{1}, \\
& V_{R}(0)=80 \varepsilon,
\end{aligned}
$$

also we have

$$
\begin{aligned}
& V_{R}(1)=\frac{-40[1(1-1)-2 \varepsilon]}{1+1}, \\
& V_{R}(1)=\frac{-40(-2 \varepsilon)}{2} \\
& V_{R}(1)=40 \varepsilon
\end{aligned}
$$

we have,

$$
\begin{aligned}
& W(x)=-\varepsilon\left[\frac{(1+x)}{\varepsilon}\right], \\
& W(x)=-(1+x),
\end{aligned}
$$

hence Eq.(2.11) becomes,

$$
V_{l}(x)=\exp \left(-\int_{0}^{x} \sqrt{(1+x) / \varepsilon} d s\right) .
$$

The first initial-value problem is given by

$$
\begin{aligned}
& V_{1}^{\prime}(x)=\left(\frac{-1}{\sqrt{\varepsilon}}\right) \sqrt{(1+x)} V_{1}(x), \\
& V_{1}(0)=1
\end{aligned}
$$

now Eq.(2.12) becomes

$$
V_{2}(x)=\exp \left(-\int_{x}^{1} \sqrt{(1+x) / \varepsilon} d s\right) .
$$

The second initial-value problem is given by

$$
\begin{aligned}
& V_{2}^{\prime}(x)=\frac{1}{\sqrt{\varepsilon}} \sqrt{(1+x)} V_{2}(x), \\
& V_{2}(1)=1 .
\end{aligned}
$$


Table 3. The maximum absolute errors for example 3.2 for $\varepsilon=10^{-4}, h=10^{-3}$.

\begin{tabular}{|c|c|c|c|}
\hline$x$ & $y(x)$ & Exact solution & Max. Absolute error \\
\hline 0.0000 & 0.0000000 & 0.0000000 & 0.0000000 \\
\hline 0.0010 & 0.0399411 & 0.0399600 & 0.0000189 \\
\hline 0.0020 & 0.0798040 & 0.0798400 & 0.0000360 \\
\hline 0.0030 & 0.1195886 & 0.1196400 & 0.0000514 \\
\hline 0.0040 & 0.1592947 & 0.1593600 & 0.0000779 \\
\hline 0.0050 & 0.1989221 & 0.1990000 & 0.0001694 \\
\hline 0.0200 & 0.7838306 & 0.7840000 & 0.0001888 \\
\hline 0.0400 & 1.5358110 & 1.5360000 & 0.0001881 \\
\hline 0.0600 & 2.2558120 & 2.2560000 & 0.0001853 \\
\hline 0.0800 & 2.9438150 & 2.9440000 & 0.0001819 \\
\hline 0.1000 & 3.5998180 & 3.6000000 & 0.0001669 \\
\hline 0.2000 & 6.3998330 & 6.4000000 & 0.0001431 \\
\hline 0.4000 & 9.5998570 & 9.6000000 & 0.0001249 \\
\hline 0.6000 & 9.5998740 & 9.5999990 & 0.0001111 \\
\hline 0.8000 & 6.3998890 & 6.4000000 & 0.0000000 \\
\hline 1.0000 & 0.0000000 & 0.0000000 & \\
\hline
\end{tabular}

Table 4.The maximum absolute errors for example 3.2 for $\varepsilon=10^{-5}, h=10^{-3}$.

\begin{tabular}{|c|c|c|c|}
\hline$x$ & $y(x)$ & Exact solution & Max. Absolute error \\
\hline 0.0000 & 0.0000000 & 0.0000000 & 0.0000000 \\
\hline 0.0010 & 0.0399546 & 0.0399600 & 0.0000054 \\
\hline 0.0020 & 0.0798307 & 0.0798400 & 0.0000093 \\
\hline 0.0030 & 0.1196278 & 0.1196400 & 0.0000122 \\
\hline 0.0040 & 0.1593457 & 0.1593600 & 0.0000158 \\
\hline 0.0050 & 0.1989842 & 0.1990000 & 0.0000196 \\
\hline 0.0200 & 0.7839805 & 0.7840000 & 0.0000193 \\
\hline 0.0400 & 1.5359810 & 1.5360000 & 0.0000188 \\
\hline 0.0600 & 2.2559810 & 2.2560000 & 0.0000186 \\
\hline 0.0800 & 2.9439820 & 2.9440000 & 0.0000184 \\
\hline 0.1000 & 3.5999820 & 3.6000000 & 0.0000167 \\
\hline 0.2000 & 6.3999830 & 6.4000000 & 0.0000143 \\
\hline 0.4000 & 9.5999860 & 9.6000000 & 0.0000124 \\
\hline 0.6000 & 9.5999870 & 9.5999990 & 0.0000110 \\
\hline 0.8000 & 6.3999890 & 6.4000000 & 0.0000000 \\
\hline 1.0000 & 0.0000000 & 0.0000000 & \\
\hline
\end{tabular}

\section{Examples 3.3}

Consider the following singular perturbation problem

$$
\begin{aligned}
& -\varepsilon\left(y^{\prime}(x)\right)^{\prime}+(1+x(1-x)) y(x)=[1+x(1-x)]+ \\
& +\left[2 \sqrt{\varepsilon}-x^{2}(1-x)\right] \exp \left(-\frac{1-x}{\sqrt{x}}\right)+\left[2 \sqrt{\varepsilon}-x(1-x)^{2}\right] \exp \left(-\frac{x}{\sqrt{\varepsilon}}\right)
\end{aligned}
$$


having boundary conditions

$$
\begin{aligned}
& y(0)=0, \\
& y(1)=0,
\end{aligned}
$$

which has the exact solution

$$
y(x)=1+(x-1) \exp \left(-\frac{x}{\sqrt{\varepsilon}}\right)-x \exp \left(-\frac{1-x}{\sqrt{x}}\right)
$$

Eq.(3.34) becomes

$$
\begin{array}{ll} 
& -\varepsilon y^{\prime \prime}(x)+(1+x(1-x)) y(x)=[1+x(1-x)]+ \\
& +\left[2 \sqrt{\varepsilon}-x^{2}(1-x)\right] \exp \left(-\frac{1-x}{\sqrt{x}}\right)+\left[2 \sqrt{\varepsilon}-x(1-x)^{2}\right] \exp \left(-\frac{x}{\sqrt{\varepsilon}}\right), \\
\text { i.e., } \quad & y^{\prime \prime}(x)-\frac{(1+x(1-x))}{\varepsilon} y(x)=-\frac{[1+x(1-x)]}{\varepsilon}+ \\
& -\frac{\left[2 \sqrt{\varepsilon}-x^{2}(1-x)\right]}{\varepsilon} \exp \left(-\frac{1-x}{\sqrt{x}}\right)-\frac{\left[2 \sqrt{\varepsilon}-x(1-x)^{2}\right]}{\varepsilon} \exp \left(-\frac{x}{\sqrt{\varepsilon}}\right),
\end{array}
$$

comparing Eqs (2.1) and (2.3), we have

$$
\begin{aligned}
& a(x)=1 \\
& b(x)=1+x(1-x) \\
& f(x)=[1+x(1-x)]+\left[2 \sqrt{\varepsilon}-x^{2}(1-x)\right] \exp \left(-\frac{1-x}{\sqrt{x}}\right)+\left[2 \sqrt{\varepsilon}-x(1-x)^{2}\right] \exp \left(-\frac{x}{\sqrt{\varepsilon}}\right),(3.3 \\
& P(x)=0 \\
& Q(x)=\frac{-[1+x(1-x)]}{\varepsilon} \\
& R(x)=\frac{[1+x(1-x)]+\left[2 \sqrt{\varepsilon}-x^{2}(1-x)\right] \exp \left(-\frac{1-x}{\sqrt{x}}\right)+\left[2 \sqrt{\varepsilon}-x(1-x)^{2}\right] \exp \left(-\frac{x}{\sqrt{\varepsilon}}\right)}{\varepsilon}
\end{aligned}
$$

also we have

$$
\begin{aligned}
& A(x)=\frac{-[1+x(1-x)]}{\varepsilon}-\frac{1}{2}(0)-\frac{1}{4}(0), \\
& A(x)=\frac{-[1+x(1-x)]}{\varepsilon},
\end{aligned}
$$


Eq.(2.7) becomes

$$
\begin{aligned}
& H(x)=R(x) \exp \left(\frac{1}{2} \int_{0}^{x}(0) d s\right) \\
& =R(x) e^{0}
\end{aligned}
$$

i.e., $H(x)=R(x)$

$$
H(x)=\frac{[1+x(1-x)]+\left[2 \sqrt{\varepsilon}-x^{2}(1-x)\right] \exp \left(-\frac{1-x}{\sqrt{x}}\right)+\left[2 \sqrt{\varepsilon}-x(1-x)^{2}\right] \exp \left(-\frac{x}{\sqrt{\varepsilon}}\right)}{\varepsilon},
$$

as Eq.(2.6) becomes

$$
\begin{aligned}
& U(x)=\exp \left(-\frac{1}{2} \int_{0}^{x}(0) d s\right), \\
& U(x)=e^{0} \\
& U(x)=1
\end{aligned}
$$

we have

$$
\begin{aligned}
& V(0)=0, \\
& V(1)=0
\end{aligned}
$$

Eq.(3.36) can be written as

$$
\begin{aligned}
& -\varepsilon V^{\prime \prime}(x)+(1+x(1-x)) V(x)=[1+x(1-x)]+ \\
& +\left[2 \sqrt{\varepsilon}-x^{2}(1-x)\right] \exp \left(-\frac{1-x}{\sqrt{x}}\right)+\left[2 \sqrt{\varepsilon}-x(1-x)^{2}\right] \exp \left(-\frac{x}{\sqrt{\varepsilon}}\right),
\end{aligned}
$$

with boundary conditions

$$
\begin{aligned}
& V(0)=0 \\
& V(1)=0,
\end{aligned}
$$

as $\varepsilon \rightarrow 0$ Eq.(3.42) becomes

$$
\begin{aligned}
& (1+x(1-x)) V_{R}(x)=[1+x(1-x)]+\left[2 \sqrt{\varepsilon}-x^{2}(1-x)\right] \exp \left(-\frac{1-x}{\sqrt{x}}\right)+ \\
& +\left[2 \sqrt{\varepsilon}-x(1-x)^{2}\right] \exp \left(-\frac{x}{\sqrt{\varepsilon}}\right),
\end{aligned}
$$




$$
V_{R}(x)=\frac{[1+x(1-x)]+\left[2 \sqrt{\varepsilon}-x^{2}(1-x)\right] \exp \left(-\frac{1-x}{\sqrt{x}}\right)+\left[2 \sqrt{\varepsilon}-x(1-x)^{2}\right] \exp \left(-\frac{x}{\sqrt{\varepsilon}}\right)}{(1+x(1-x))}
$$

hence

$$
\begin{aligned}
& V_{R}(0)=\left(1+2 \sqrt{\varepsilon} \exp \left[\frac{-1}{\sqrt{\varepsilon}}\right]+2 \sqrt{\varepsilon}\right), \\
& V_{R}(1)=\left[1+2 \sqrt{\varepsilon}+2 \sqrt{\varepsilon} \exp \left[\frac{-1}{\sqrt{\varepsilon}}\right]\right],
\end{aligned}
$$

we have,

$$
\begin{aligned}
& W(x)=-\varepsilon\left[\frac{-[1+x(1-x)]}{\varepsilon}\right], \\
& W(x)=1+x(1-x),
\end{aligned}
$$

Eq.(2.11) leads to

$$
\begin{aligned}
& V_{l}(x)=\exp \left(-\int_{0}^{x} \sqrt{-(1+x(1-x)) / \varepsilon} d s\right), \\
& V_{l}^{\prime}(x)=\exp \left(-\int_{0}^{x} \sqrt{-(1+x(1-x)) / \varepsilon} d s\right)\left(\sqrt{\frac{-1}{\varepsilon}(1+x(1-x))}\right) .
\end{aligned}
$$

The first initial value problem is given by

$$
\begin{aligned}
& V_{1}^{\prime}(x)=V_{l}(x)\left(\sqrt{\frac{-1}{\varepsilon}(1+x(1-x))}\right), \\
& V_{1}(0)=1 .
\end{aligned}
$$

Now Eq.(2.12) becomes

$$
\begin{aligned}
& V_{2}(x)=\exp \left(-\int_{x}^{1} \sqrt{-(1+x(1-x)) / \varepsilon} d s\right), \\
& V_{2}^{\prime}(x)=\exp \left(-\int_{x}^{1} \sqrt{-(1+x(1-x)) / \varepsilon} d s\right)[\sqrt{((1+x(1-x)) / \varepsilon)}] .
\end{aligned}
$$

The second initial-value problem given by

$$
V_{2}^{\prime}(x)=\frac{1}{\sqrt{\varepsilon}} V_{2}(x)[\sqrt{(1+x(1-x))}]
$$

with

$$
V_{2}(1)=1
$$


Table 5. The maximum absolute errors for example 3.3 for $\varepsilon=10^{-4}, h=10^{-3}$.

\begin{tabular}{|c|c|c|c|}
\hline$x$ & $y(x)$ & Exact solution & Max. Absolute error \\
\hline 0.0000 & 0.0000000 & 0.0000000 & 0.0000000 \\
\hline 0.0010 & 0.0944957 & 0.0960674 & 0.0015717 \\
\hline 0.0020 & 0.1801083 & 0.1829067 & 0.0027984 \\
\hline 0.0030 & 0.2576682 & 0.2614042 & 0.0037360 \\
\hline 0.0040 & 0.3279287 & 0.3323613 & 0.0044325 \\
\hline 0.0050 & 0.3915730 & 0.3965020 & 0.0049290 \\
\hline 0.0200 & 0.8640755 & 0.8673714 & 0.0032959 \\
\hline 0.0400 & 0.9818980 & 0.9824170 & 0.0005190 \\
\hline 0.0600 & 0.9976332 & 0.9976700 & 0.0000368 \\
\hline 0.0800 & 0.9996957 & 0.9996914 & 0.0000043 \\
\hline 0.1000 & 0.9999614 & 0.9999591 & 0.0000023 \\
\hline 0.2000 & 1.0000000 & 1.0000000 & 0.0000000 \\
\hline 0.4000 & 1.0000000 & 1.0000000 & 0.0000000 \\
\hline 0.6000 & 1.0000000 & 1.0000000 & 0.0000000 \\
\hline 0.8000 & 1.0000000 & 1.0000000 & 0.0000000 \\
\hline 1.0000 & 0.0000000 & 0.0000000 & 0.0000000 \\
\hline
\end{tabular}

Table 6. The maximum absolute errors for example 3.3 for $\varepsilon=10^{-5}, h=10^{-3}$.

\begin{tabular}{|c|c|c|c|}
\hline$x$ & $y(x)$ & Exact solution & Max. Absolute error \\
\hline 0.0000 & 0.0000000 & 0.0000000 & 0.0000000 \\
\hline 0.0010 & 0.2705911 & 0.2718355 & 0.0012444 \\
\hline 0.0020 & 0.4680499 & 0.4697770 & 0.0017271 \\
\hline 0.0030 & 0.6121177 & 0.6139112 & 0.0017934 \\
\hline 0.0040 & 0.7172136 & 0.7188647 & 0.0016511 \\
\hline 0.0050 & 0.7938672 & 0.7952881 & 0.0014209 \\
\hline 0.0200 & 0.9982367 & 0.9982440 & 0.0000073 \\
\hline 0.0400 & 0.9999971 & 0.9999969 & 0.0000002 \\
\hline 0.0600 & 1.0000000 & 1.0000000 & 0.0000000 \\
\hline 0.0800 & 1.0000000 & 1.0000000 & 0.0000000 \\
\hline 0.1000 & 1.0000000 & 1.0000000 & 0.0000000 \\
\hline 0.2000 & 1.0000000 & 1.0000000 & 0.0000000 \\
\hline 0.4000 & 1.0000000 & 1.0000000 & 0.0000000 \\
\hline 0.6000 & 1.0000000 & 1.0000000 & 0.0000000 \\
\hline 0.8000 & 1.0000000 & 1.0000000 & 0.0000000 \\
\hline 1.0000 & 0.0000000 & 0.0000000 & 0.0000000 \\
\hline
\end{tabular}

\section{Conclusions}

In this paper, we have discussed the application of initial-value techniques for some model problems involving small parameter $\varepsilon$. We have first transformed the original problem into the normal form and then converted it into two initial-value problems. It is a practical method and can be easily implemented on a computer to solve such problems. Three examples are given to demonstrate the efficiency of the proposed method. The maximum absolute errors $\max _{i}\left|y\left(x_{i}\right)-y_{i}\right|$ at different nodal points are tabulated in the tables 
for $\varepsilon=10^{-4}$ and $\varepsilon=10^{-5}$. The results further corroborate the applicability of the proposed method. It has been observed that the present method approximates the exact solution very well.

\section{Nomenclature}

$$
\begin{aligned}
& \varepsilon- \text { a small positive parameter } \\
& \alpha, \beta- \text { real constants } \\
& a(x), b(x) \text { and }(x)- \text { smooth functions in }[0,1] \\
& P(x)=\frac{a^{\prime}(x)}{a(x)} \\
& Q(x)=\frac{-b(x)}{\varepsilon a(x)} \\
& R(x)=\frac{-f(x)}{\varepsilon a(x)}
\end{aligned}
$$

\section{References}

[1] O’Malley R.E. (1974): Introduction to Singular Perturbations. - New York: Academic Press.

[2] Nayfeh A.H. (1981): Introduction to Perturbation Techniques. - New York: Wiley.

[3] Kevorkian J. and Cole J.D. (1981): Perturbation Methods in Applied Mathematics. - New York: Springer-Verlag.

[4] Bender C.M. and Orszag S.A. (1978): Advanced Mathematical Methods for Scientists and Engineers. - New York: McGraw-Hill.

[5] Van Dyke M. (1964): Perturbation Methods in Fluid Mechanics. - New York: Academic Press.

[6] Wasow W. (1965): Asymptotic Expansions for Ordinary Differential Equations. - New York: Inter Science.

[7] Hemker P.W. (1977): A numerical study of stiff two point boundary problems. - MCT 80, Mathematical Centre, Amsterdam.

[8] Hemker P.W. and Miller J.J.H. (Eds.) (1979): Numerical Analysis of Singular Perturbation Problems. - New York: Academic Press.

[9] Doolan E.P., Miller J.J.H. and Schilders W.H.A. (1980): Uniform Numerical Methods for Problems with Initial and Boundary Layers. - Dublin: Boole Press.

[10] Morton K.W. (1995): Numerical Solution of Convection - Diffusion Problems. - Oxford University Press.

[11] Gasparo M.G. and Macconi M. (1990): Initial value methods for second-order singularly perturbed boundaryvalue problems. - Journal of Optimization Theory and Applications, vol.66, pp.197-210.

[12] Gasparo M.G. and Macconi M. (1989): New initial-value method for singularly perturbed boundary-value problems. - Journal of Optimization Theory and Applications, vol.63, pp.213-224.

[13] Gasparo M.G. and Macconi M. (1992): Numerical solution of second-order nonlinear singularly perturbed boundary-value problems by initial value methods. - Journal of Optimization Theory and Applications, vol.73, pp.309-327.

[14] Gasparo M.G. and Macconi M. (1992): Parallel initial-value algorithms for singularly perturbed boundary-value problems. - Journal of Optimization Theory and Applications, vol.73 , pp.501-517.

[15] Reddy Y.N. and PramodChakravarthy P. (2003): Method of Reduction of Order for Solving Singularly Perturbed Two-Point Boundary Value Problems. - Applied Mathematics and Computation,vol.136, pp 27-45.

[16] Reddy Y.N. and PramodChakravarthy P. (2004): An initial-value approach for solving singularly perturbed twopoint boundary value problem.- Applied Mathematics and Computation,vol.155, pp.95-110. 
[17] Mishra H.K., Kumar M. and Singh P. (2009): Initial value technique for self adjoint singular perturbation boundary value problems. - Computational Mathematics and modeling, vol.29, No.2.

[18] Natesan S. and Ramanujam N. (1998): Initial-value technique for singularly perturbed boundary value problems for second-order ordinary differential equations arising in chemical reactor theory. - Journal of Optimization Theory and Applications, vol.97, No.2, pp.455-470.

[19] Kadalbajoo M.K. and Reddy Y.N. (1987): An initial value technique for a class of non-linear singular perturbation problems. - Journal of Optimization Theory and Applications, vol.53, pp.395-406.

[20] Pearson C.E. (1968): On a differential equation of boundary layer type. - J. Math. Phys., vol.47, pp.134-154.

Received: January 31, 2019

Revised: November 27, 2019 